\title{
An evaluation of distal hair cortisol concentrations collected at delivery
}

\section{( 野 Article in press ? )}

- Orta, O.R. ${ }^{\mathrm{E} E m a i l}$ Author,

- Tworoger, S.S.ab,

- Terry, K.L. ac,

- Coull, B.A. ${ }^{a}$,

- Gelaye, B. ${ }^{a}$,

- Kirschbaum, C. ${ }^{d}$,

- Sanchez, S.E.ef,

- Williams, M.A. ${ }^{a}$

- View Correspondence (jump link)

- $\quad$ aHarvard T.H. Chan School of Public Health, Harvard University, MA, USA

- $\quad$ bH. Lee Moffitt Cancer Center \& Research Institute, Tampa, FL, USA

- 'Brigham and Women's Hospital and Harvard Medical School, Boston, MA, USA

- $\quad$ TTechnische Universität Dresden, Dresden, Germany

- eFacultad de Medicina, Universidad Peruana de Ciencias Aplicadas (UPC), Lima, Peru

- $\quad$ 'Asociacion Civil Proyectos en Salud (PROESA), Lima, Peru

Hide additional affiliations_

\begin{abstract}
Distal hair segments collected at delivery may allow for the assessment of maternal cortisol secretion in early pregnancy, an important time window for fetal development. Therefore, an investigation of the validity of distal hair cortisol concentrations is warranted. We examined the concordance between proximal and distal hair cortisol concentrations ( $\mathrm{HCC}$ ), both representing the first trimester of pregnancy. The study population was comprised of a random sample of 97 women participating in the Pregnancy Outcomes Maternal and Infant Study, a prospective cohort study of pregnant women attending prenatal clinics in Lima, Peru. Each participant provided two hair samples: once at enrollment [mean gestational age $(G A)=13.1$ weeks] and again at full-term delivery (mean GA = 39.0 weeks). Hair segments reflecting the first trimester were: $3 \mathrm{~cm}$ hair segments closest to the scalp on the first hair sample (proximal) and 6-9 cm from the scalp on the second hair sample (distal). HCC was determined using Luminescence Immunoassay. A subset $(\mathrm{N}=28)$ had both hair segments additionally analyzed using liquid chromatography tandem mass spectrometry (LC-MS/MS). HCC values were log-transformed (logHCC), and proximal-distal differences tested using paired sample t-tests. Concordance was evaluated within and across assay types. LogHCC, measured using immunoassay, in distal hair segments was lower compared to proximal hair
\end{abstract}


segments (1.35 versus 1.64 respectively; $p=.02)$. No difference was observed using LC-MS/MS (1.99 versus 1.83, respectively; $p=.33$ ). Proximal-distal concordance was low within assay (immunoassay: Pearson $=0.27$ and $\mathrm{k}=0.10$; LC-MS/MS:

Pearson $=0.37$ and $k=0.07$ ). High correlation was observed across assays for both distal (Pearson $=0.78, p<.001 ; \mathrm{k}=0.64)$ and proximal segments (Pearson $=0.96$, $p<.001 ; k=0.75)$. In conclusion, distal first-trimester hair segments collected at delivery have lower absolute $\mathrm{HCC}$ compared to $\mathrm{HCC}$ in proximal first trimester hair segments collected in early pregnancy, and are poorly concordant with HCC in proximal segments. Findings may inform the design of future studies. (c) 2018 Informa UK Limited, trading as Taylor \& Francis Group

Author keywords

\section{Cortisolhairpregnancystress}

- ISSN: 10253890

- CODEN: STREF

- Source Type: Journal

- Original language: English

- DOI: $10.1080 / 10253890.2018 .1458088$

- Document Type: Article in Press

- Publisher: Taylor and Francis Ltd 